\title{
The secondary prevention of hepatitis B virus- associated hepatocellular carcinoma
}

\author{
Bo-Bin Hü, Rong-Ming Wang", Jian-Ning Jiang \\ Department of Infectious Diseases, The First Affiliated Hospital of Guangxi Medical University, Nanning 530021, Guangxi, China. \\ "Authors contributed equally.
}

Correspondence to: Dr. Jian-Ning Jiang, Department of Infectious Diseases, The First Affiliated Hospital of Guangxi Medical University, No.6, Shuangyong Road, Nanning 530021, Guangxi, China. E-mail: jjianning@163.com

How to cite this article: Hu BB, Wang RM, Jiang JN. The secondary prevention of hepatitis B virus-associated hepatocellular carcinoma. Hepatoma Res 2019:5:29. http://dx.doi.org/10.20517/2394-5079.2019.23

Received: 26 Jun 2019 First Decision: 27 Jun 2019 Revised: 28 Jun 2019 Accepted: 11 Jul 2019 Published: 7 Aug 2019

Science Editor: Guang-Wen Cao Copy Editor: Lan Ma Production Editor: Cai-Hong Wang

Primary liver cancer can be classified into three categories according to different pathological types: hepatocellular carcinoma (HCC), intrahepatic cholangiocarcinoma (ICC), and combined HCC-ICC. Among them, HCC accounts for more than $85 \%-90 \%$. Therefore, the term "liver cancer" in this article refers specifically to HCC. Due to an insidious onset and no symptoms in the early stage of HCC, as well as lacking of awareness of disease screening of patients with HCC, they go to hospital only when the symptoms are vivid and diagnose with advanced HCC with a survival period of three to six months. For this reason, HCC was once known as "the king of cancer". Clinically, we have noticed that most patients with HCC have a natural history of acute hepatitis B virus (HBV) infection - chronic hepatitis B (CHB) - liver cirrhosis (LC) - HCC, which shows that HBV infection is closely related to LC and HCC. According to the statistics, more than two billion people worldwide have been infected with HBV, and 240 million of them are CHB. Over 650 thousand people die every year from liver failure, LC and HCC caused by HBV, and $60 \%$ LC and $80 \%$ HCC are HBV-related ${ }^{[1]}$. The major hazard of HBV infection is considered to be chronic infection, which plays an important role in hepatocellular carcinogenesis. Hepatic fibrosis and LC are susceptible in CHB, which may eventually lead to the occurrence of HCC.

Since the implementation of planned immunization in China, the number of CHB patients has dropped steadily, while the stock of that is still large. For the carrying rate of hepatitis B surface antigen ( $\mathrm{HBs} A g)$ in general population is estimated to be $7.18 \%$, it is calculated that about 93 million people are chronically infected with HBV, about 20 million of whom are CHB patients. According to the natural history mentioned above, if these people do not receive whole course management and standard treatment, nearly 700 thousand people will develop into HCC in 8-10 years later.

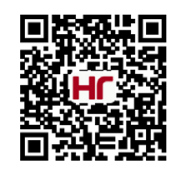


There may be two possible modes of HBV carcinogenesis: one is viral oncogene mode, that is, after the body is infected with HBV, HBV will integrate into the host genome by incorporating its own genes into the nucleus of hepatic cells, thus transforming normal hepatic cells into cancer cells ${ }^{[2-4]}$; the other is the cellular oncogene mode, that is, viral DNA will integrate into or rearranges with DNA of its host to produce HBVactivated genes or proto-oncogenes, leading to the transformation of normal cells into cancer cells and ultimately the development of $\mathrm{HCC}^{[5,6]}$.

It is not impossible to prevent HCC. If we can take intervention measures at the stage of CHB to prevent the disease progression to a great extent, it is impossible to prevent a considerable number of patients with CHB from developing into LC or even HCC. This article will focus on the secondary prevention of HBVrelated HCC-early detection, early diagnosis, standard antiviral treatment, and whole-course management - to show its importance and clinical significance.

Screening of high-risk groups of HBV-associated HCC is helpful for early detection and diagnosis. Currently, it is known that the high risk factors closely lead to the progression of HCC in patients with CHB mainly include: hepatitis B e antigen ( $\mathrm{HBe} \mathrm{Ag}$ ) positive, genotype C HBV, high HBV DNA load, long-term intake of a lot of alcohol, people with LC basis and a family history of HCC, especially men over 40 years old and so on. Regular physical examinations should be carried out in high risk population. Regular examination of liver function, HBV serological tests, HBV-DNA load, AFP, abdominal ultrasound, and non-invasive liver fibrosis detection and the like every three to six months, enhanced CT test or nuclear magnetic resonance (MRI) should be further examined in suspected patients, in order to detect small HCC early. Early surgical resection and other radical treatment can improve the cure rate of $\mathrm{HCC}$ and prolong the life cycle of patients as far as possible.

Standard antiviral treatment plays a crucial role in secondary prevention of HBV-associated HCC. In patients with $\mathrm{CHB}$, antiviral treatment has been shown to prevent disease progression to LC and $\mathrm{HCC}^{[7-11]}$. A retrospective cohort study showed ${ }^{[12]}$ that after 5 years of follow-up, the cumulative incidence of HCC in nucleotide analogue (NUCs) antiviral treatment group and control group (without treatment) was 3.7\%-13.7\%, while the cumulative incidence of HCC was 7\%-38.9\% in these two groups of HBV-related LC (HBLC) patients, indicating a long-term of antiviral treatment can reduce the risk of HCC significantly. Similar studies also suggested ${ }^{[13]}$ that long-term NUCs treatment was associated with 77\% reduction of HCC risk in LC patients. Our team has been working on a cohort study of long-term standardized antiviral treatment and whole-course management for patients with CHB. Our previous studies showed ${ }^{[14]}$ that compared with the control groups, the cumulative incidence of LC in CHB patients treated with NUCs for 3-5 years was $1.4 \%$ vs. $10.2 \%$ and $2.7 \%$ vs. $22.4 \%$, respectively $(P<0.001)$, the cumulative incidence of HCC in $3-5$ years was $0.2 \% v s .2 .3 \%$ and $0.9 \%$ vs. $3.4 \%$, respectively $(P=0.017)$ [Figure 1]. All the results mentioned above illustrated that long-term and standard antiviral treatment can significantly reduce the risk of LC and HCC in patients with CHB.

Thus, a whole course management should be conducted in patients with high risk of HCC starting from the discovery of HBsAg positive, including chronic HBV carriers, non-active HBsAg carriers, HBeAg-positive and negative CHB patients, and HBLC patients in compensated and decompensated periods. As for the chronic HBV carriers and non-active HBsAg carriers, blood routine, biochemistry, HBV serological tests, AFP and the like should be monitored every 3 months, abdominal ultrasound, computed tomography (CT) or non-invasive liver fibrosis detection should be carried out every 6 months, and liver biopsy should be conducted if it is necessary. If antiviral treatment indications are met, treatment should be started in time. For HBeAg-positive CHB patients, after 3-6 months of observation, antiviral treatment could be started if alanine aminotransferase level continued to rise and there was no spontaneous HBeAg serological conversion. On the contrary, for $\mathrm{HBeAg}$-negative $\mathrm{CHB}$ patients, antiviral treatment should be started as soon as possible if they meet the indication. For patients with HBLC, antiviral treatment should be initiated immediately [Figure 2]. After starting antiviral treatment, patients should be followed up regularly by telephone, text 


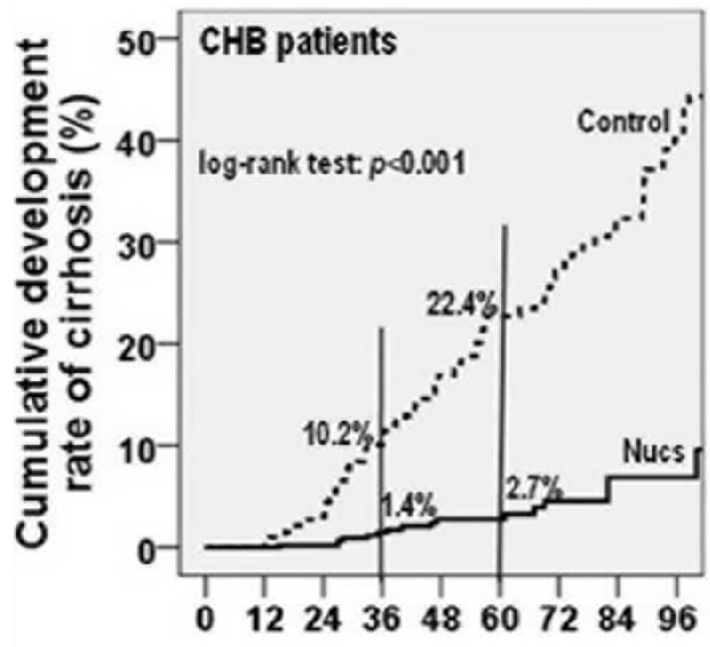

No.at risk Follow-up months

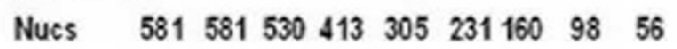

Control $447 \quad 447 \quad 375 \quad 254 \quad 177 \quad 133 \quad 106 \quad 88 \quad 72$

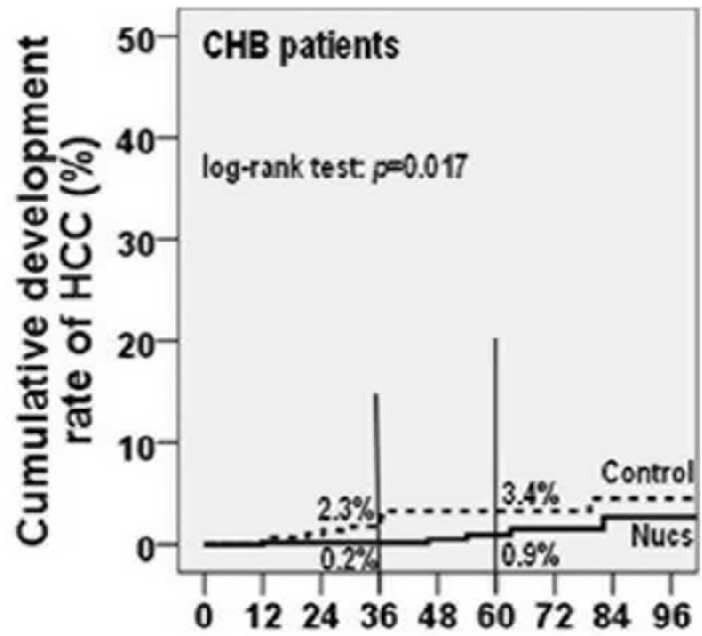

No.at risk Follow-up months

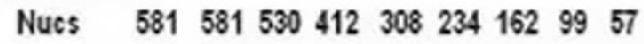

Control $447 \quad 447372 \quad 246 \quad 172128103 \quad 85 \quad 67$

Figure 1. Clinical outcomes of long-term NUCs treatment

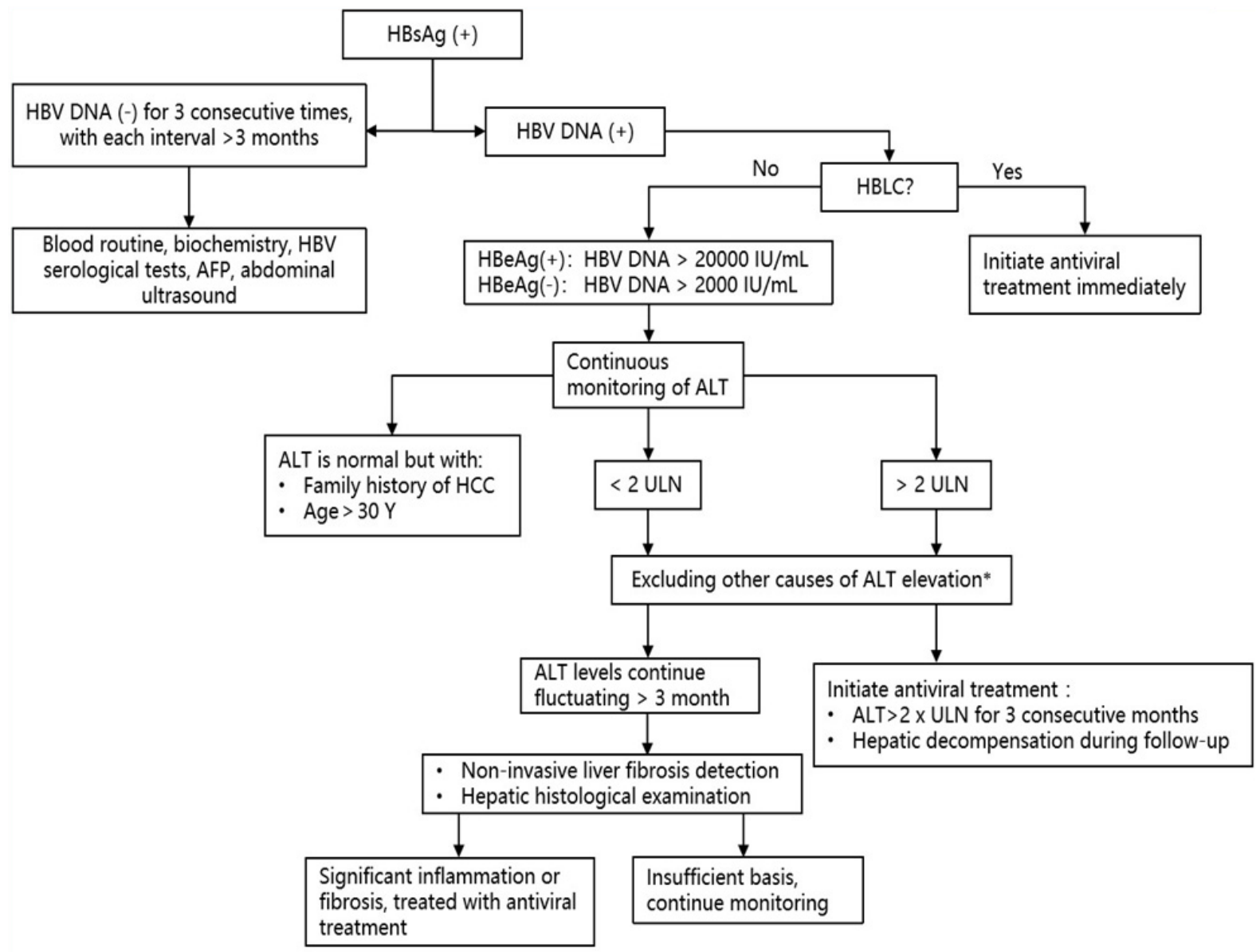

* Other common causes of elevated ALT: other pathogenic infections, drugs, alcohol, immunity, fatty liver, etc.

Figure 2. Indications of antiviral treatment (2015 China CHB prevention and treatment guidelines) 


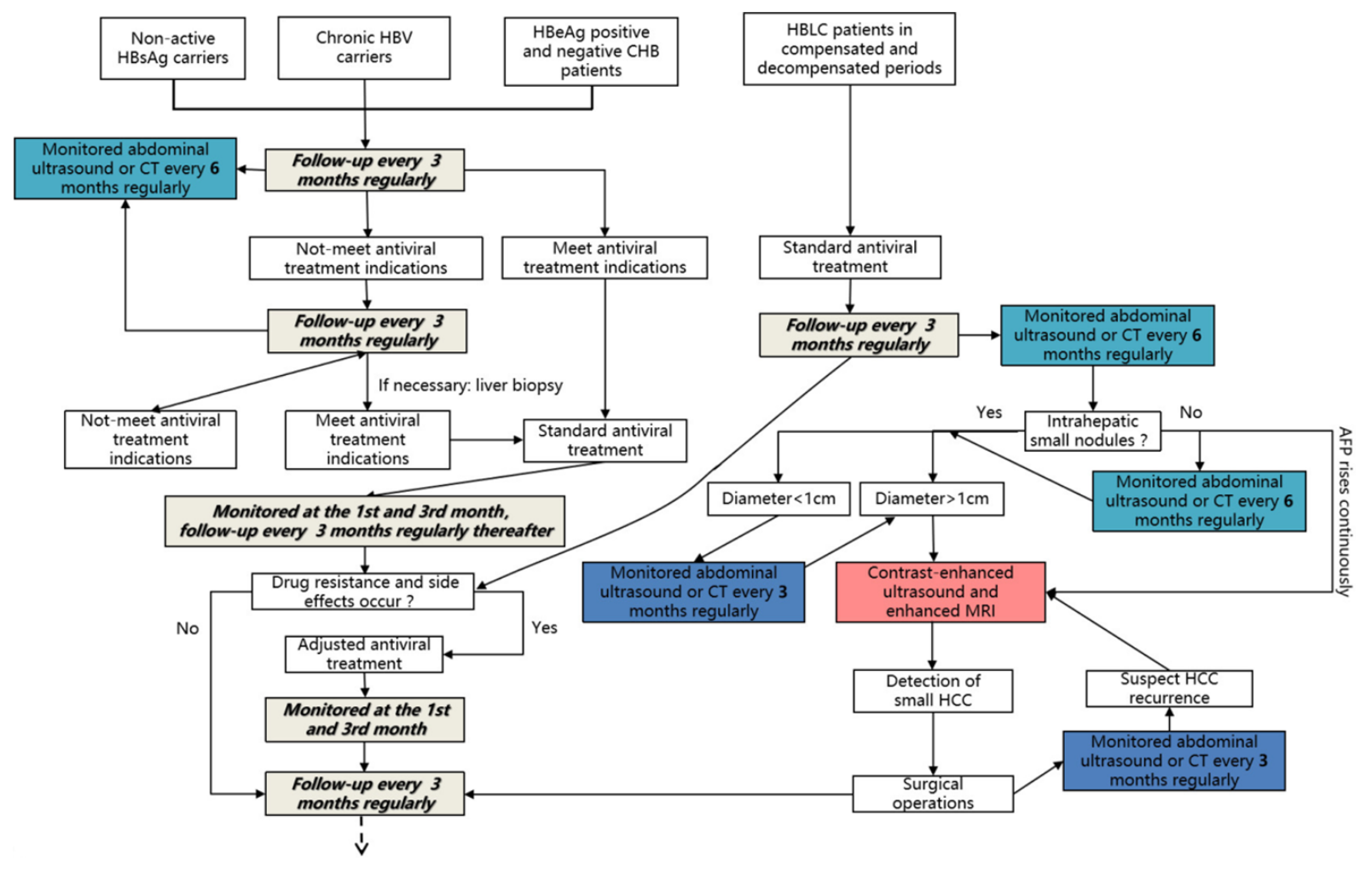

Figure 3. Whole-course management-no end in sight

message, email, WeChat and other methods. Patients with hepatitis B cirrhosis should be reminded to examine HBV-DNA load, HBV serological tests, liver function, AFP and the like every 3 months. Abdominal ultrasound or CT test should be carried out every 6 months. Once the intrahepatic small nodules are found, and if the diameter of the nodules is larger than $1 \mathrm{~cm}$, contrast-enhanced ultrasound and enhanced MRI should be performed to determine whether it is a small liver cancer. Once the diagnosis of small liver cancer is confirmed, surgical operation should be performed immediately. However, if the diameter of the nodules is smaller than $1 \mathrm{~cm}$ and unable to confirm diagnosis by imaging examination, closely followup should be performed instead, including monitoring abdominal ultrasound or CT test every 3 months. Once the intrahepatic small nodules are found to become bigger progressively, take intervene according to the above procedure. If no small intrahepatic nodules are found by imaging examination, abdominal ultrasound or CT test should be continued monitoring every 6 months. Once intrahepatic small nodules are found, interventions should be actively taken according to the above procedure. Patients with small liver cancers which are found early, after surgical operation, should be reminded monitoring abdominal ultrasound or CT test every 3 months. Once there is a possibility of recurrence of liver cancer, contrastenhanced ultrasound and enhanced MRI should be performed, and surgical intervention should be taken immediately after diagnosis. Regular follow-up should be continued after surgery, repeatedly. Treatment plans should be adjusted in time once drug resistance and side effects occur during the follow-up period, and the biochemical and virological examinations should be performed in the 1st and 3rd months after the adjustment of the therapy, and continue to maintain regular follow-up every 3 months thereafter. Doctorpatient interaction should be advocated to supervise patients to continue taking antiviral drugs if they were found to stop taking medicines. At the same time, patients should be educated on the knowledge of hepatitis $\mathrm{B}$ to improve their understanding of the disease [Figure 3]. Through all the managements mentioned above, in our follow-up cohort, about $70 \%$ of HCC patients were found only with small HCC, and all of them received timely surgical resection and treatment, and continued to follow up postoperatively, so as to achieve the goal of early detection, early diagnosis, and early treatment. 
Secondary prevention of HBV-associated HCC plays a key role in reducing the incidence of HCC, finding the occurrence of small HCC, carrying out early surgical treatments, and prolonging the survival cycle of patients, which deserved to be widely popularized.

\section{DECLARATIONS}

\section{Authors' contributions}

Collected the data and drew figures: $\mathrm{Hu} B \mathrm{~B}$

Drafted the manuscript: Wang RM

Obtained the funding and revised the paper: Jiang JN

\section{Availability of data and materials}

Not applicable.

\section{Financial support and sponsorship}

This work was supported by grants from Natural Science Foundation of Guangxi Key Laboratory for the Prevention and Control of Viral Hepatitis (GXCDCKL201802) and was funded by Key laboratory of HighIncidence-Tumor Prevention \& Treatment (Guangxi Medical University), Ministry of Education (GKE2018-05), and National Science and Technology Major Project of China (2017ZX10202201).

\section{Conflicts of interest}

All authors declared that there are no conflicts of interest.

\section{Ethical approval and consent to participate}

Not applicable.

\section{Consent for publication}

Not applicable.

\section{Copyright}

(c) The Author(s) 2019.

\section{REFERENCES}

1. Hou JL, Lai W; Chinese Society of Hepatology, Chinese Medical Association; Chinese Society of Infectious Diseases, Chinese Medical Association. The guideline of prevention and treatment for chronic hepatitis B: a 2015 update. Zhonghua Gan Zang Bing Za Zhi 2015;23:888-905. (in Chinese)

2. Tsai WL, Chung RT. Viral hepatocarcinogenesis. Oncogene 2010;29:2309-24.

3. Saitta C, Tripodi G, Barbera A, Bertuccio A, Smedile A, et al. Hepatitis B virus (HBV) DNA integration in patients with occult HBV infection and hepatocellular carcinoma. Liver Int 2015;35:2311-7.

4. Kawai-Kitahata F, Asahina Y, Tanaka S, Kakinuma S, Murakawa M, et al. Comprehensive analyses of mutations and hepatitis B virus integration in hepatocellular carcinoma with clinicopathological features. J Gastroenterol 2016;51:473-86.

5. Lau CC, Sun T, Ching AK, He M, Li JW, et al. Viral-human chimeric transcript predisposes risk to liver cancer development and progression. Cancer Cell 2014;25:335-49.

6. Liang HW, Wang N, Wang Y, Wang F, Fu Z, et al. Hepatitis B virus-human chimeric transcript HBx-LINE1 promotes hepatic injury via sequestering cellular microRNA-122. J Hepatol 2016;64:278-91.

7. European Association for the Study of the Liver. EASL clinical practice guidelines: management of hepatocellular carcinoma. J Hepatol 2018;69:182-236.

8. Sung JJ, Tsoi KK, Wong VW, Li KC, Chan HL. Meta-analysis: treatment of hepatitis B infection reduces risk of hepatocellular carcinoma. Aliment Pharmacol Ther 2008;28:1067-77.

9. Papatheodoridis G V, Manolakopoulos S, Touloumi G, Vourli G, Raptopoulou-Gigi M, et al. Virological suppression does not prevent the development of hepatocellular carcinoma in $\mathrm{HBeAg}$-negative chronic hepatitis B patients with cirrhosis receiving oral antiviral(s) starting with lamivudine monotherapy: results of the nationwide HEPNET. Greece cohort study. Gut 2011;60:1109-16.

10. Lai CL, Yuen MF. Prevention of hepatitis B virus-related hepatocellular carcinoma with antiviral therapy. Hepatology 2013;57:399-408.

11. Yang F, Liu WB, Chen L, Tan XJ, Cao GW, et al. Anti-HBV treatment delays development and evolution of HCC. Academic Journal of 
Second Military Medical University 2014,35.

12. Tetsuya Hosaka, Antiviral Drug Cuts Cancer Risk in Hepatitis B, medpagetoday, November 12, 2012.

13. Reduction of hepatocellular carcinoma in hepatitis B-related cirrhosis patients with long-term entecavir therapy - A follow-up report of C-TEAM study. AASLD2014. LB-30.

14. Jiang JN, Li SH, Su MH, Zhong SH, Wang BJ, et al. The clinical outcomes of the CHB and LC patient treated with long term nucleos(t) ide analogs under whole-course management: a real-life cohort study. J Hepatol 2014;60:S361-522. 